\title{
BRIEF
}

\section{Survey of Pass/Fail Grading Systems in US Doctor of Pharmacy Curricula}

\author{
Joel P. Spiess, MS, Erin Walcheske, MS, George E. MacKinnon III, PhD, MS, Karen J. MacKinnon, BPharm
}

Medical College of Wisconsin, School of Pharmacy, Milwaukee, Wisconsin

Corresponding Author: Joel P. Spiess, Medical College of Wisconsin, School of Pharmacy, 8701 Watertown Plank Rd., Milwaukee, WI 53226. Tel: 414-955-2858. Email: jspiess@ mcw.edu

Submitted December 21, 2020; accepted May 28, 2021; ePublished June 2021

Objective. To understand how colleges and schools of pharmacy utilize pass/fail grading systems in Doctor of Pharmacy curricula.

Methods. An electronic survey with 15 selected response items and six open-ended questions was developed and distributed in 2020 to the 10 academic pharmacy programs known to utilize a pass/fail grading system to gather qualitative and quantitative data.

Results. Leaders from eight of the 10 programs identified responded to the survey. Programs varied regarding the types of courses for which they utilize a pass/fail grading system and whether numerical scores are shared with students. A variety of grade designations (honors, pass, no pass, fail, satisfactory, etc.) are used, and the minimum pass level varies by program, ranging from $70 \%$ to $90 \%$. For those institutions that utilize post-course remediation, the majority of remediation occurs immediately following the academic term or in the summer. The type of information shared with residency program directors (GPA, class rank, overall percentile, qualitative comments, etc.) varies among pass/fail programs.

Conclusion. How pass/fail grading systems are utilized is inconsistent across schools and colleges pharmacy. Programs that utilize a criterion-based grading system might benefit from engaging in future conversations with one another to determine if and how consistency might be realized among terminology, passing level, percentages, grade point averages, and progression. Additional insights on post-graduate training requirements and honorary societies is warranted should pass/fail grading expand as it has in medical education. Further research on this topic may lead to greater understanding and adoption across the pharmacy academy.

Keywords: pass/fail, grading, assessment, pharmacy student, residency

\section{INTRODUCTION}

The evidence behind pass/fail grading systems suggests benefits to students, including increased well-being, cohort cohesion, motivation, and the encouragement of self-regulated learning and decreased anxiety and stress and competition within cohorts. ${ }^{1-4}$ Furthermore, studies by Miller and Spring and colleagues have found that pass/fail grading systems do not, by default, negatively impact academic performance. ${ }^{5,6}$ These benefits are exemplified by the fact that the majority of medical schools employ such a grading system; however, widespread adoption of a pass/fail grading system has not occurred within the pharmacy academy. ${ }^{7}$

Many programs in academic medicine have migrated toward grading systems that have attempted to relieve student stress, anxiety, and competitiveness while enhancing cooperative learning among students. ${ }^{8}$ Such attempts are intended to enhance the overall psychological well-being of the student body. In a multi-institutional study by Reed and colleagues that surveyed over 2,000 first- and second-year medical students at seven US medical schools, it was concluded that "curriculum reform intended to enhance student well-being should incorporate pass/fail grading." At present well-over three-quarters of medical schools utilize a form of a pass/fail grading system in the four years of education. $^{7}$

This study sought to better understand how Doctor of Pharmacy (PharmD) programs utilize this approach to grading, including the types of courses for which the pass/fail model is used, whether numerical scores or grade point averages (GPA) are shared with students, when remediation occurs, and what type of information is shared with residency programs, employers, and honor societies. The authors were also interested in learning how information and data, if any, are communicated to pharmacy residency program directors to ensure students without a GPA or class rank are competitive (or even permitted to apply).

\section{METHODS}


Based on firsthand knowledge and a review of program websites, the authors first assembled a list of those colleges and schools of pharmacy known to be using a pass/fail grading system for their PharmD programs. This list was shared with and affirmed by members of the American Association of Colleges of Pharmacy (AACP) Curriculum Special Interest Group via AACP Connect, the association's online discussion forum. ${ }^{9}$ The authors then developed a survey to gather qualitative and quantitative data from those 10 programs identified. The survey was pre-tested by the authors but not by any external stakeholders. The pharmacy deans and associate deans of each of the 10 institutions were invited to participate via email with a customized link, so only one response per institution was obtained. The instrument contained 15 selected response questions and six open-ended questions for a total of 21 questions, with logic, so the total number of questions varied depending on responses. The survey was divided into four sections: institutional information, pass/fail practices, student communication, and residency information. The survey was distributed using Qualtrics (Qualtrics LLC, Provo, Utah) in May 2020 and three reminders were sent out over six weeks. The study was approved by the Medical College of Wisconsin (Milwaukee, Wisconsin) Institutional Review Board.

The survey responses were interpreted using Qualtrics reports due to the limited sample size and the descriptive nature of the data. The qualitative data was used to support the selected response questions to provide more clarity rather than to identify themes. Information on the institutions, including length of program, curricular delivery, and enrollment was collected as part of the survey and reviewed. The researchers also reviewed questions on pass/fail grading, remediation strategies, and how information is communicated to residency program directors.

\section{RESULTS}

Eight academic pharmacy leaders from the 10 schools (80\%) are represented in the data set. From those, half were from public institutions and half were from private institutions. The majority (63\%) were from three-year programs. The curricular delivery was split between block (38\%), quarter (38\%) and semester (25\%). Total enrollment for the most recently matriculated group of students ranged from 49 to 340, with an average of 129 students.

All eight institutions utilized a pass/fail grading system on introductory pharmacy practice experience (IPPE) courses, and the majority (88\%) utilized the system on didactic, elective, lab, advanced pharmacy practice experience (APPE), and IPE courses. Only 63\% and 50\% utilized this system on co-curricular courses/activities and experiential education seminars, respectively.

Institutions utilized various grade terminology within pass/fail grading system including Honors (63\%), Pass (63\%), No Pass (13\%), Fail (50\%), Satisfactory (38\%), Unsatisfactory (25\%) and Competency (13\%). Additionally, the minimum pass level also varied by institution, ranging from $70 \%$ to $90 \%$. Institutions also varied on when, and in what types of courses, grades as a numerical value (percentages) are released to students, as depicted in Table 1.

Institutions utilized a variety of remediation strategies, including skills remediation in labs (88\%), post-course remediation (88\%), re-examinations (75\%), and skills remediation on rotations $(50 \%)$. For those institutions that utilized a post-course remediation, the majority of remediation occurs immediately following the academic term or in the summer and rarely occurs in another academic term concurrent with or in place of new coursework. The majority of institutions (75\%) stated that students cannot progress without pausing if they earn a final grade below passing. However, each program has its own remediation and dismissal policies that it follows based on individual student cases.

Only one institution calculates a GPA for its students based on the final numerical score in the class. Other institutions provide a class rank, overall percentile, or qualitative comments from APPE preceptors. Some utilize the final percentage score in the course to equate to a typical letter grade system and award quality points accordingly. Other programs award the maximum quality points available to each student who earns a passing grade in the course - described by one survey respondent as "meaningless." Finally, two institutions indicated use of a course average percentage calculation or class rank to determine participation in Rho Chi.

\section{DISCUSSION}

The results suggest that PharmD programs that utilize a pass/fail grading system do so quite differently from other programs using a similar approach to student assessment. This is true across various types of programs, be they public or private or three or four years in duration. Future work to address the following areas may lead to increased understanding and incorporation of this grading system across the pharmacy academy.

1. Terminology. Among the eight programs that responded to the survey, the terminology utilized to describe student achievement levels (grade designations) varies. Academic programs could work together to unify the language they use to describe performance levels to establish benchmarking purposes and help faculty, students, preceptors, residency program directors, and other employers become more familiar with how to interpret such information.

2. Passing Level. Across the programs surveyed, the variance among the minimum pass level for a course was great, ranging from $70 \%$ to $90 \%$. One program reported that the pass level for each course was determined by the course 
director. A different program reported that the program utilizes a minimum pass level of $70 \%$ but that individual courses may choose a higher minimum competency level based upon the content of the course. Some programs also reported that their minimum pass level has already changed, or they are considering changes for the future.

3. Scores Released to Students. Programs vary regarding whether they display percentages to students on assignments and assessments and for the overall course grade before the pass/fail term is assigned. There may be consideration given to whether the type of the course (eg, didactic, skill, experiential) should influence whether or not a percentage should be displayed to the student. For example, if the passing score for a didactic course is $75 \%$, and the student sees only "Pass" for an exam grade, the student may not know if they barely passed at $75 \%$ or if they mastered nearly all of the material and earned a $94 \%$.

4. Grade Point Average. While most PharmD programs surveyed do not calculate a GPA, class rank, or overall numerical percentile, some do. The authors hypothesize that those that do calculate GPA likely do so to be able to provide such information to residency programs and honor societies such as Rho Chi. Those pass/fail programs that calculate a numeric score also vary in their method of doing so as described in the Results section. Those programs that do not calculate a numeric score for their students each take different approaches to educating residency programs and employers about their grading system and individual student performance.

5. Progression. A primary concern of all academic pharmacy programs is student progression. While some students may experience academic difficulty along the way to earning the PharmD degree, the impact of an academic failure can vary among programs that utilize a pass/fail grading system. While programs with a traditional letter grade system may permit a maximum number of " $\mathrm{D}$ " grades in the program, pass/fail programs must determine if it is appropriate for a student to progress and/or graduate with a "Fail" grade in a course that is not repeated. Furthermore, the outcome of a failing grade can be significantly influenced by other factors, such as the academic calendar and whether or not the program allows for adequate time between terms for students to complete remediation - a unique challenge for accelerated programs.

In medical education, advancements have not ceased with the wide-spread adoption of pass/failing grading systems in various facets of education and training. In February 2020, it was announced that there would be a movement away from a numerical score on the US Medical Licensing Examination (USMLE) Step I to pass/fail, beginning in 2022. ${ }^{10}$ This was promulgated by the overwhelming desire to improve student well-being and that the USMLE examinations (Step I and II) were not developed for evaluation of student academic achievement but rather licensure. ${ }^{11}$ There were additional reasons for this change. When USMLE Step I scores are utilized for screening medical residency applicants, Black, Hispanic, and females are offered fewer interviews and residency positions. ${ }^{12}$

In December 2020, citing alignment with the current practice of other professional licensure board exams and reporting practices, the National Association of Boards of Pharmacy (NABP) announced that both the North American Pharmacist Licensure Examination and the Multistate Pharmacy Jurisprudence Examination would move to a pass/fail result beginning in January 2021. ${ }^{13}$

Given the prevailing movement in medical education to pass/fail grading systems, as well as the NABP's changes to licensure exam scoring, why has academic pharmacy not embraced such a movement? While this question cannot be answered fully by this study, it certainly is worthy of a conversation in the academy and across the profession as it looks to enhance student pharmacists' psychological well-being in all aspects of their education and training.

It should be noted that the small number of programs that utilize a pass/fail grading system is a limitation of this study and the potential implications and future directions outlined above. Additionally, the authors cannot be certain that all schools with this assessment model were surveyed; thus, the need for some level of ongoing assessment and conversation in the academy on this topic is warranted.

\section{CONCLUSION}

A pass/fail grading system is known to be utilized by 10 PharmD programs in the US. This project surveyed these programs to gather key information to better understand approaches to this grading system. The results indicated a variety of approaches are utilized, which may lead to confusion and lack of adoption within the pharmacy academy. This study suggests that programs that utilize a pass/fail grading system might benefit greatly from engaging in future conversations with one another to determine if and how consistency might be realized across institutions. The primary items to consider include terminology, passing level, percentages, GPAs, and progression. As more discussion in academic pharmacy ensues around student wellness, competencies-driven curricula, entrustable professional activities, and criterion-based grading, movement towards pass/fail grading systems is part of this conversation. Such work described here lays the foundation for a robust discussion only likely to grow. 


\section{REFERENCES}

1. Babal JC, Abraham O, Webber S, Watterson T, Moua P, Chen J. Student Pharmacist Perspectives on Factors That Influence Wellbeing During Pharmacy School. Am J Pharm Educ. 2020;84(9). doi:10.5688/ajpe7831

2. Brazeau GA, Frenzel JE, Prescott WA. Facilitating Wellbeing in a Turbulent Time. Am J Pharm Educ. 2020;84(6):ajpe8154. doi:10.5688/ajpe8154

3. Reed DA, Shanafelt TD, Satele DW, et al. Relationship of Pass/Fail Grading and Curriculum Structure With Well-Being Among Preclinical Medical Students: A Multi-Institutional Study: Acad Med. 2011;86(11):13671373. doi:10.1097/ACM.0b013e3182305d81

4. Ip EJ, Nguyen K, Shah BM, Doroudgar S, Bidwal MK. Motivations and Predictors of Cheating in Pharmacy School. Am J Pharm Educ. 2016;80(8):133. doi:10.5688/ajpe808133

5. Miller BM. Can a Pass/Fail Grading System Adequately Reflect Student Progress? AMA J Ethics. 2009;11(11):842-851. doi:10.1001/virtualmentor.2009.11.11.ccas2-0911

6. Spring L, Robillard D, Gehlbach L, Moore Simas TA. Impact of pass/fail grading on medical students' well-being and academic outcomes: Impact of pass/fail grading on student outcomes. Med Educ. 2011;45(9):867-877. doi:10.1111/j.1365-2923.2011.03989.x

7. $\quad$ Grading Systems Use by US Medical Schools. LCME Annual Medical School Questionnaire Part II, 2014-2015 through 2018-2019. https://www.aamc.org/data-reports/curriculum-reports/interactive-data/grading-systems-useus-medical-schools

8. Bloodgood RA, Short JG, Jackson JM, Martindale JR. A Change to Pass/Fail Grading in the First Two Years at One Medical School Results in Improved Psychological Well-Being: Acad Med. 2009;84(5):655-662. doi:10.1097/ACM.0b013e31819f6d78

9. Spiess J. Pass/Fail Doctor of Pharmacy Programs. AACP Connect. Published January 15, 2020. https://connect.aacp.org/communities/groupdetails/digestviewer/viewthread?MessageKey=5b9ac988-1ccb-4c96b24a-2fd3371701a4\&CommunityKey=332efba0-6aca-4284-9c213996b261b67c\&tab=digestviewer\&Token=C8D3DDCF-3786-451A-864A-739F4BBE3F0F\#bm5b9ac988-1ccb4c96-b24a-2fd3371701a4

10. MacKinnon GE, Payne S, Drolet BC, Motuzas C. Pass/Fail USMLE Step 1 Scoring-A Radiology Program Director Survey. Acad Radiol. Published online September 2020:S1076633220304918. doi:10.1016/j.acra.2020.08.010

11. Whelan AJ. The Change to Pass/Fail Scoring for Step 1 in the Context of COVID-19: Implications for the Transition to Residency Process. Acad Med. 2020;95(9):1305-1307. doi:10.1097/ACM.0000000000003449

12. Rubright JD, Jodoin M, Barone MA. Examining Demographics, Prior Academic Performance, and United States Medical Licensing Examination Scores: Acad Med. 2019;94(3):364-370. doi:10.1097/ACM.0000000000002366

13. Garrity M. NABP Examination Program Changes. Published online December 10, 2020. 
Table 1. Percentage of Surveyed Institutions Releasing Grades as Percentage Scores to Students by Course Type

\begin{tabular}{lcccc}
\hline & $\begin{array}{c}\text { Each } \\
\text { Assignment } \\
\mathbf{N = 8 ( \% )}\end{array}$ & $\begin{array}{c}\text { Each } \\
\text { Assessment }^{\text {a }} \\
(\mathbf{\%})\end{array}$ & $\begin{array}{c}\text { Midterm } \\
\text { Course Grade } \\
(\mathbf{\%})\end{array}$ & $\begin{array}{c}\text { Final } \\
\text { Course Grade } \\
(\boldsymbol{\%})\end{array}$ \\
\hline Didactic course & 88 & 88 & 75 & 50 \\
Skills or lab course & 63 & 75 & 50 & 38 \\
Experiential course & 25 & 38 & 63 & 50 \\
\hline
\end{tabular}

${ }^{a}$ Assessment was defined as exam/quiz in the survey. 